\title{
Case Report \\ Proximal Limb Weakness in a Patient with Celiac Disease: Copper Deficiency, Gluten Sensitivity, or Both as the Underlying Cause?
}

\author{
J. David Avila ${ }^{1,2}$ and David Lacomis ${ }^{2,3}$ \\ ${ }^{1}$ Department of Neurology, Geisinger Medical Center, Danville, PA, USA \\ ${ }^{2}$ Department of Neurology, University of Pittsburgh Medical Center, Pittsburgh, PA, USA \\ ${ }^{3}$ Department of Pathology, University of Pittsburgh Medical Center, Pittsburgh, PA, USA \\ Correspondence should be addressed to J. David Avila; avila.jd83@gmail.com
}

Received 29 August 2016; Revised 20 October 2016; Accepted 2 November 2016

Academic Editor: Pablo Mir

Copyright (C) 2016 J. D. Avila and D. Lacomis. This is an open access article distributed under the Creative Commons Attribution License, which permits unrestricted use, distribution, and reproduction in any medium, provided the original work is properly cited.

Celiac disease has been associated with several neurologic disorders which may result from micronutrient deficiencies, coexisting autoimmune conditions, or gluten sensitivity. Copper deficiency can produce multiple neurologic manifestations. Myeloneuropathy is the most common neurologic syndrome and it is often irreversible, despite copper replacement. We report the case of a 55year-old man who presented with progressive proximal limb weakness and weight loss in the setting of untreated celiac disease without gastrointestinal symptoms. He had anemia, neutropenia, and severe hypocupremia. The pattern of weakness raised the suspicion that there was an underlying myopathy, although this was not confirmed by electrodiagnostic studies. Weakness and hematologic abnormalities resolved completely within 1 month of total parenteral nutrition with copper supplementation and a gluten-free diet. Myopathy can rarely occur in patients with celiac disease, but the mechanism is unclear. Pure proximal limb weakness has not been previously reported in copper deficiency. We propose that this may represent a novel manifestation of hypocupremia and recommend considering copper deficiency and gluten sensitivity in patients presenting with proximal limb weakness.

\section{Introduction}

Celiac disease (CD) is a systemic autoimmune condition triggered by dietary gluten in genetically susceptible individuals. It has a prevalence of 0.6 to $1 \%$ worldwide [1]. Although historically considered a gastrointestinal disorder, approximately half of patients present with extraintestinal symptoms [1]. Several neurologic disorders have been associated with CD [2-4]. Whether they result from micronutrient deficiencies, coexisting autoimmune disorders, or gluten sensitivity alone is not entirely clear [5].

Copper deficiency may occur in the setting of zinc poisoning, gastric bypass surgery, and malabsorption syndromes. It can manifest with multiple hematologic and neurologic abnormalities. Anemia and neutropenia are the most common hematologic derangements [6]. Among the neurologic manifestations myeloneuropathy and ataxic myelopa- thy, similar to subacute combined degeneration, have been well described [7]. Other less typical presentations include motor neuron disease, optic neuropathy, and central nervous system demyelination [8-10]. To our knowledge, pure proximal limb weakness has not been previously reported.

Herein, we describe a patient with copper deficiency and $\mathrm{CD}$ who presented with symmetric proximal limb weakness and had complete resolution of symptoms with copper replacement and gluten-free diet.

\section{Case Report}

A 55-year-old man with a previous history of peripheral vascular disease, $C D$, and hypothyroidism presented with 4 months of bilateral leg weakness and weight loss. He was a retired police officer and had been physically very strong in the past. $\mathrm{CD}$ was diagnosed elsewhere 5 years earlier. 
TABLE 1: Laboratory findings at diagnosis and after 1 month of TPN.

\begin{tabular}{lccc}
\hline Parameter & Baseline & After 1 month of TPN & Normal range \\
\hline RBC $\left(\times 10^{12} / \mathrm{L}\right)$ & $\mathbf{3 . 8 2}$ & 4.42 & $4.13-5.57$ \\
Hemoglobin $(\mathrm{g} / \mathrm{L})$ & $\mathbf{1 2 . 5}$ & 14.8 & $12.9-16.9$ \\
$\mathrm{MCV}(\mathrm{fL})$ & $\mathbf{1 0 2 . 3}$ & $\mathbf{9 9 . 3}$ & $82.6-97.4$ \\
WBC $\left(\times 10^{9} / \mathrm{L}\right)$ & $\mathbf{2 . 6}$ & 6.7 & $3.8-10.6$ \\
ANC $\left(\times 10^{9} / \mathrm{L}\right)$ & $\mathbf{0 . 5}$ & 2.1 & $1.5-7.7$ \\
Platelets $\left(\times 10^{9} / \mathrm{L}\right)$ & 369 & 293 & $156-369$ \\
Copper $(\mu \mathrm{g} / \mathrm{dL})$ & $\mathbf{6}$ & 93 & $60-190$ \\
Ceruloplasmin $(\mathrm{mg} / \mathrm{dL})$ & $\mathbf{3 . 1}$ & - & $22-58$ \\
Vitamin $\mathrm{B}_{12}(\mathrm{pg} / \mathrm{mL})$ & $\mathbf{1 9 3}$ & 811 & $211-911$ \\
Zinc $(\mu \mathrm{g} / \mathrm{dL})$ & 0.72 & 0.78 & $0.55-1.5$ \\
Vitamin D $(\mathrm{ng} / \mathrm{mL})$ & 32 & - & $30-50$ \\
Vitamin E $(\mu \mathrm{g} / \mathrm{mL})$ & 8 & - & $5.5-17$ \\
Anti-gliadin Ab IgA $(\mathrm{AU})$ & $\mathbf{1 6 5}$ & $\mathbf{1 3 7}$ & $<20$ \\
Anti-gliadin Ab IgG $(\mathrm{AU})$ & $\mathbf{3 9}$ & $\mathbf{3 5}$ & $<20$ \\
Anti-TTG IgA (units) & $\mathbf{1 1 0}$ & $\mathbf{6 4}$ & $<20$ \\
\hline
\end{tabular}

TPN: total parenteral nutrition; RBC: red blood cells; MCV: mean corpuscular volume; WBC: white blood cells; ANC: absolute neutrophil count; Ab: antibody; AU: arbitrary units; TTG: tissue transglutaminase.

The details of his initial evaluation were not available. The disease was thought to be inactive and the patient was on an unrestricted diet.

The symptoms were slowly progressive. He had difficulty rising from a chair and going up stairs. He denied eyelid ptosis, diplopia, dysarthria, dysphagia, dyspnea, myalgia, fasciculations, muscle atrophy, numbness, paresthesia, and bladder or bowel problems. He had unintentional weight loss of $30 \mathrm{Lb}$. There was no loss of appetite, decreased food intake, diarrhea, or steatorrhea. Examination demonstrated 4/5 strength in the deltoids and iliopsoas bilaterally (Medical Research Council scale). There was no weakness of intermediate and distal limb muscles. Cranial nerves, reflexes, sensation, coordination, and gait were normal.

He had a 60-pack-year history of smoking and the initial investigations were focused on a possible malignancy. Laboratory workup was remarkable for macrocytic anemia and neutropenia (Table 1). Peripheral blood smear and flow cytometry showed no evidence of leukemia or lymphoma. Bone marrow biopsy demonstrated hypocellularity with no other abnormalities. Computed tomography of the chest, abdomen, and pelvis was normal. Acetylcholine receptor and voltage-gated calcium channel antibodies were negative. Serum creatine kinase and thyroid function tests were also normal. Electrodiagnostic studies (EDX) were performed per our myopathy protocol, which includes one motor and one sensory nerve conduction study (NCS) from an arm and a leg (Ulnar, tibial, and sural NCS in this case), and needle examination of multiple proximal and distal arm and leg muscles as well as a thoracic paraspinal muscle. Additionally, we performed $3 \mathrm{~Hz}$ repetitive nerve stimulation of the ulnar, accessory, and facial nerves. There were no abnormalities found on EDX. Further testing revealed severe copper deficiency [6 $\mu \mathrm{g} / \mathrm{dL}(60-190)]$ and mild $\mathrm{B}_{12}$ deficiency [193 pg/mL (211-911)], as well as positive anti-gliadin and anti-

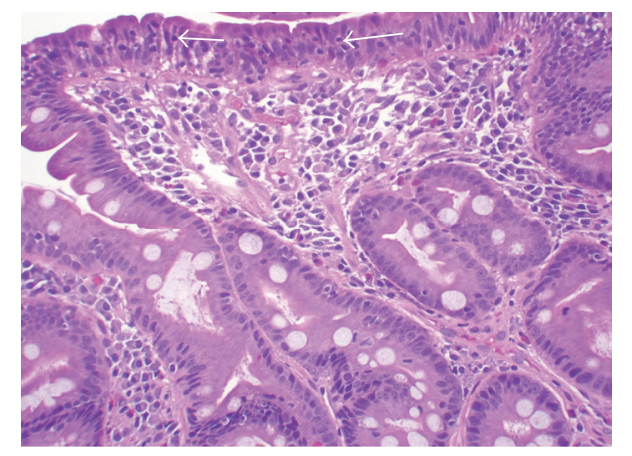

FIGURE 1: Duodenal biopsy specimen showing villous blunting and intraepithelial lymphocytosis (white arrows). There is also chronic inflammation in the lamina propria and crypt hyperplasia consistent with gluten sensitive enteropathy (hematoxylin and eosin stain).

transglutaminase antibodies. Zinc and vitamins D and $\mathrm{E}$ were normal (Table 1).

The patient underwent endoscopy and duodenal biopsy that showed moderate villous blunting, intraepithelial lymphocytosis, chronic inflammation of the lamina propria, and crypt hyperplasia. The histologic findings, in conjunction with the elevated anti-transglutaminase antibodies, confirmed the diagnosis of active gluten sensitive enteropathy or CD (Figure 1). He was placed on a strict gluten-free diet and total parenteral nutrition (TPN) with micronutrient supplementation. The daily dose of copper was $2 \mathrm{mg}$. He also had weekly $B_{12}$ intramuscular injections.

After 1 month of TPN, copper and other micronutrients normalized (Table 1). Anemia and neutropenia improved. Weakness resolved completely and the patient regained $10 \mathrm{Lb}$. TPN was stopped and he remained on a gluten-free diet. 


\section{Discussion}

Neurologic manifestations have been reported in $8 \%$ of patients with $\mathrm{CD}$ [2]. They may be present in individuals with or without enteropathy, as defined by an abnormal small bowel biopsy, and may be the initial presentation of the disease [3]. The most common manifestations are cerebellar ataxia, polyneuropathy, and encephalopathy [3]. Neurologic deficits are attributed to $\mathrm{CD}$ or gluten sensitivity when there is no alternative explanation and symptoms improve with a gluten-free diet $[3,4]$.

Different micronutrient deficiencies may occur as a consequence of malabsorption in CD. Interestingly, micronutrient deficiencies can occur in the absence of gastrointestinal symptoms [11]. Copper deficiency may be present in up to $15 \%$ of patients with CD [11]. Myeloneuropathy is the most common neurologic syndrome in this scenario and it is often irreversible [12]. Other nutritional deficiencies include vitamin $B_{12}$, folate, vitamin $D$, and vitamin $E$, all of which can lead to neurologic complications.

There are conflicting reports in the literature regarding the cause of neurologic disorders in CD. A study of 68 patients with neurologic dysfunction and $\mathrm{CD}$ or anti-gliadin antibody positivity found that the majority $(57 \%)$ had a discernible cause for the deficits, different from gluten exposure [5]. Coexisting autoimmunity was the most common (19\%), followed by malabsorption-induced nutritional deficiency (10\%). Most patients deteriorated, despite a gluten-free diet [5]. This study suggested that alternative causes for neurologic dysfunction should be sought in $\mathrm{CD}$ and that further therapy may be required, in addition to dietary restriction. On the contrary, a large retrospective study of 562 patients with neurologic dysfunction and $\mathrm{CD}$ or nonceliac gluten sensitivity (i.e., no enteropathy) reported that all patients responded to a gluten-free diet, regardless of the severity and type of dysfunction [3]. The authors stated that no alternative etiology for the deficits was found, despite extensive investigations. The details of those investigations, however, were not provided.

Our patient presented with symmetric proximal limb weakness, which is the most common pattern of weakness in myopathies [13]. Even though EDX did not confirm a myopathic process, this was our suspicion based on the distribution of weakness and absence of sensory deficits and physical signs of other pure motor syndromes, such as motor neuron disease and neuromuscular junction disorders. Furthermore, a normal EDX does not exclude a myopathy, as several myopathic disorders may be associated with normal results, particularly the metabolic and endocrine myopathies [14]. We felt that hypocupremia and CD were the likely causes of weakness and, since we planned on treating them, we did not pursue a muscle biopsy that was likely to be of low yield unless the patient failed to respond to treatment. In retrospect, the histopathology might have shown nonspecific evidence of myopathy and support that diagnostic entity, but we still believe it was not clinically necessary.

Myopathy is rare in CD, accounting for only $4 \%$ of all neurologic manifestations [3]. It has been described in patients with vitamin $\mathrm{D}$ and $\mathrm{E}$ deficiencies accompanying
CD $[15,16]$. Hadjivassiliou et al. proposed that myopathy may represent an extraintestinal manifestation of gluten sensitivity and that it may have an immune mechanism [17]. The authors postulated that transglutaminase type 2, the target of anti-endomysial antibodies (EMA) in CD, may be implicated in the immune-mediated muscle injury, as this enzyme is normally present in the endomysial connective tissue. However, in their study of 13 patients with biopsyproven myopathy, only 2 had positive EMA [17]. Copper levels were not provided.

CD may also coexist with inflammatory myopathies, as it occurs with other autoimmune disorders $[17,18]$. In the same study by Hadjivassiliou et al., 6 of 13 patients had pathologic findings similar to polymyositis. All but one improved with immunosuppressive therapy and gluten-free diet [17]. A Spanish study evaluated the presence of antibodies associated with CD in patients with inflammatory myopathies [18]. In their cohort of 51 patients, 17 (31\%) had anti-gliadin IgA antibodies. Interestingly, the strongest association was present in patients with sporadic inclusion-body myositis (sIBM), which is known not to respond to immunosuppressive therapy. There were no patients with anti-tissue transglutaminase or anti-endomysial antibodies. Five patients underwent jejunal biopsy and 3 met criteria for enteropathy or CD. Of those, 2 had sIBM and did not respond to gluten-free diet. The other patient had dermatomyositis and had full resolution of weakness with glucocorticoids and gluten restriction [18].

We cannot definitively determine the cause of weakness in our patient. Even though he had vitamin $\mathrm{B}_{12}$ deficiency, we feel this is unlikely to explain the symptoms as the deficiency was relatively mild and it typically produces distal limb weakness and sensory loss, due to neuropathy, myelopathy, or a combination of both. Additionally, the neurology of vitamin $B_{12}$ deficiency has been studied extensively, and myopathy has not been described [19]. We consider copper deficiency a plausible culprit, given the severity of hypocupremia, absence of other nutritional deficiencies known to produce proximal limb weakness (vitamins D and E), hematologic abnormalities, and resolution of symptoms with copper replacement and gluten-free diet. If this is the case, this would represent a novel manifestation of copper deficiency, adding to the spectrum of neurologic dysfunction in hypocupremia. Alternatively, the deficits may be the result of direct gluten sensitivity, malabsorption of another, yet unidentified micronutrient, or a combination of mechanisms.

It is important to emphasize that the patient did not have hyperzincemia. Zinc interferes with the intestinal absorption of copper and increases its sequestration in enterocytes by inducing the expression of metallothioneins, which have higher affinity for copper than zinc [20]. Zinc toxicity is a common precipitant of copper deficiency, and it may result from excessive or prolonged intake of zinc supplements and use of zinc-containing denture fixative $[7,8,10]$. In patients with hypocupremia, a normal zinc level should raise clinical suspicion for an underlying gastrointestinal pathology and trigger investigations for malabsorption syndromes, including $\mathrm{CD}$.

This case report has two main limitations. First, we could not definitely localize the clinical deficits to the muscle or 
the nervous system. Second, we are unable to prove with certainty that copper deficiency was the cause of weakness, as stated before. However, this is our speculation based on the aforementioned reasons. In the future, assessing copper levels in patients with $\mathrm{CD}$ and myopathy may clarify this issue.

We recommend considering copper deficiency and gluten sensitivity in patients presenting with proximal limb weakness, even in the absence of clinically overt enteropathy. Additional evidence is required to determine if this manifestation truly represents a myopathy. Copper replacement, in addition to gluten restriction, may lead to complete resolution of symptoms, as occurred in this case.

\section{Disclosure}

This case was presented in poster form at the 61st Annual Meeting of the American Association of Neuromuscular and Electrodiagnostic Medicine, October 29-November 1, 2014, Savannah, Georgia.

\section{Competing Interests}

The authors declare that there are no competing interests regarding the publication of this paper.

\section{References}

[1] A. Fasano and C. Catassi, "Clinical practice. Celiac disease," The New England Journal of Medicine, vol. 367, no. 25, pp. 2419-2426, 2012.

[2] W. T. Cooke and W. T. Smith, "Neurological disorders associated with adult coeliac disease," Brain, vol. 89, no. 4, pp. 683722,1966

[3] M. Hadjivassiliou, D. G. Rao, R. A. Grìnewald et al., "Neurological dysfunction in coeliac disease and non-coeliac gluten sensitivity," The American Journal of Gastroenterology, vol. 111, pp. 561-567, 2016.

[4] G. Cicarelli, G. Della Rocca, M. Amboni et al., "Clinical and neurological abnormalities in adult celiac disease," Neurological Sciences, vol. 24, no. 5, pp. 311-317, 2003.

[5] A. McKeon, V. A. Lennon, S. J. Pittock, T. J. Kryzer, and J. Murray, "The neurologic significance of celiac disease biomarkers," Neurology, vol. 83, no. 20, pp. 1789-1796, 2014.

[6] T. R. Halfdanarson, N. Kumar, C. Y. Li et al., "Hematological manifestations of copper deficiency: a retrospective review," European Journal of Haematology, vol. 80, no. 6, pp. 523-531, 2008.

[7] N. Kumar, J. B. Gross Jr., and J. E. Ahlskog, "Copper deficiency myelopathy produces a clinical picture like subacute combined degeneration," Neurology, vol. 63, no. 1, pp. 33-39, 2004.

[8] C. C. Weihl and G. Lopate, "Motor neuron disease associated with copper deficiency," Muscle and Nerve, vol. 34, no. 6, pp. 789-793, 2006.

[9] R. T. Naismith, J. B. Shepherd, C. C. Weihl, N. T. Tutlam, and A. H. Cross, "Acute and bilateral blindness due to optic neuropathy associated with copper deficiency," Archives of Neurology, vol. 66, no. 8, pp. 1025-1027, 2009.

[10] C. I. Prodan, N. R. Holland, P. J. Wisdom, S. A. Burstein, and S. S. Bottomley, "CNS demyelination associated with copper deficiency and hyperzincemia," Neurology, vol. 59, no. 9, pp. 1453-1456, 2002.

[11] J. E. Botero-López, M. Araya, A. Parada et al., "Micronutrient deficiencies in patients with typical and atypical celiac disease," Journal of Pediatric Gastroenterology and Nutrition, vol. 53, no. 3, pp. 265-270, 2011.

[12] T. R. Halfdanarson, N. Kumar, W. J. Hogan et al., "Copper deficiency in celiac disease," Journal of Clinical Gastroenterology, vol. 43, no. 2, pp. 162-164, 2009.

[13] R. J. Barohn, M. M. Dimachkie, and C. E. Jackson, "A pattern recognition approach to patients with a suspected myopathy," Neurologic Clinics, vol. 32, no. 3, pp. 569-593, 2014.

[14] D. Lacomis, "Electrodiagnostic approach to the patient with suspected myopathy," Neurologic Clinics, vol. 30, no. 2, pp. 641660, 2012.

[15] O. Z. Karaahmet, E. Unlu, F. Karaahmet, E. Gurcay, and A. Cakci, "Myopathy related to vitamin D deficiency in patient with celiac disease," Muscle and Nerve, vol. 50, no. 1, pp. 147148, 2014.

[16] K. A. Kleopa, K. Kyriacou, E. Zamba-Papanicolaou, and T. Kyriakides, "Reversible inflammatory and vacuolar myopathy with vitamin E deficiency in celiac disease," Muscle and Nerve, vol. 31, no. 2, pp. 260-265, 2005.

[17] M. Hadjivassiliou, A. K. Chattopadhyay, R. A. Grünewald et al., "Myopathy associated with gluten sensitivity," Muscle and Nerve, vol. 35, no. 4, pp. 443-450, 2007.

[18] A. Selva-O’Callaghan, F. Casellas, I. De Torres, E. Palou, J. M. Grau-Junyent, and M. Vilardell-Tarrés, "Celiac disease and antibodies associated with celiac disease in patients with inflammatory myopathy," Muscle and Nerve, vol. 35, no. 1, pp. 49-54, 2007.

[19] E. Reynolds, "Vitamin B12, folic acid, and the nervous system," The Lancet Neurology, vol. 5, no. 11, pp. 949-960, 2006.

[20] R. J. Cousins, "Absorption, transport, and hepatic metabolism of copper and zinc: special reference to metallothionein and ceruloplasmin," Physiological Reviews, vol. 65, no. 2, pp. 238309, 1985. 


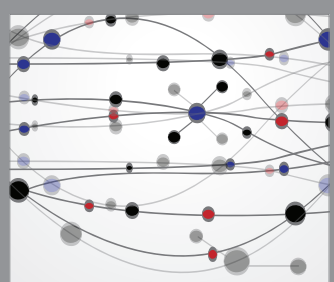

The Scientific World Journal
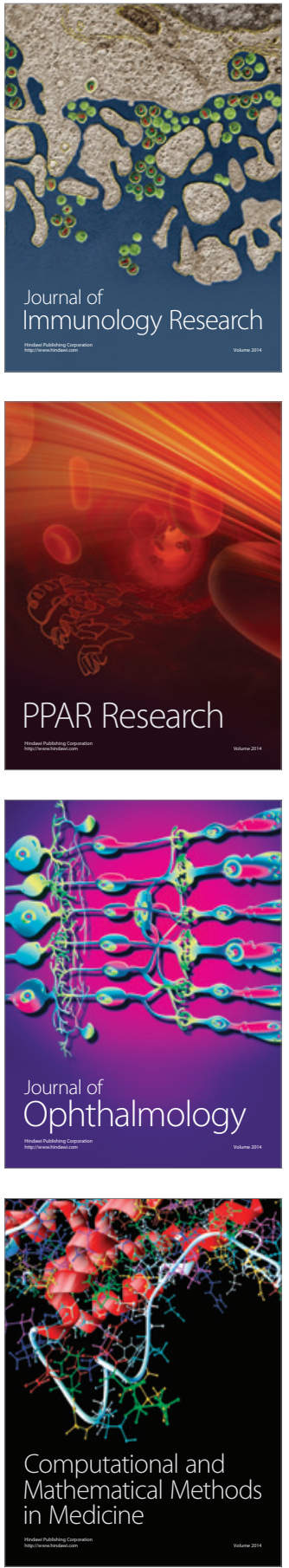

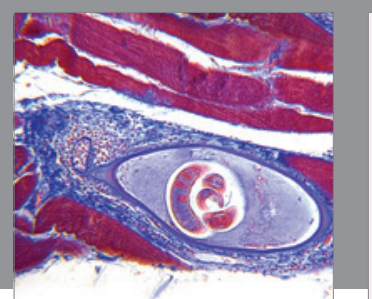

Gastroenterology Research and Practice

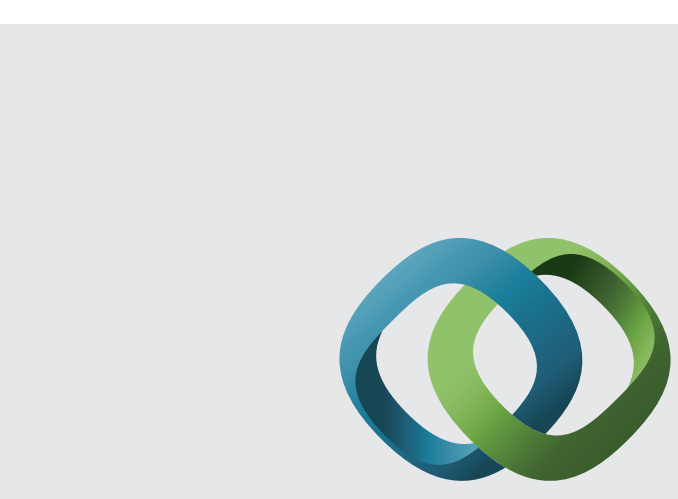

\section{Hindawi}

Submit your manuscripts at

http://www.hindawi.com
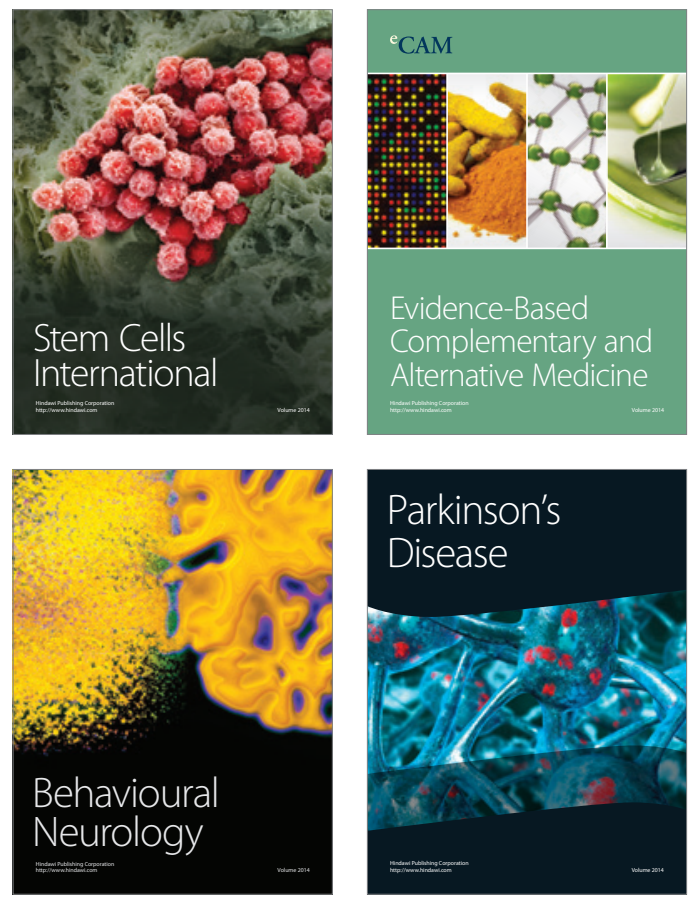
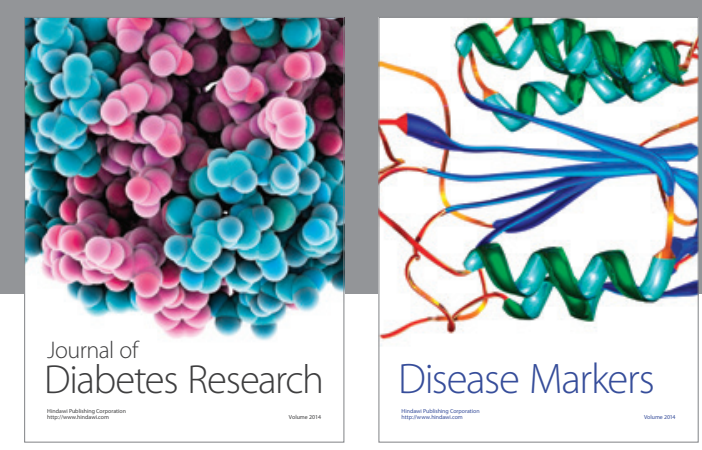

Disease Markers
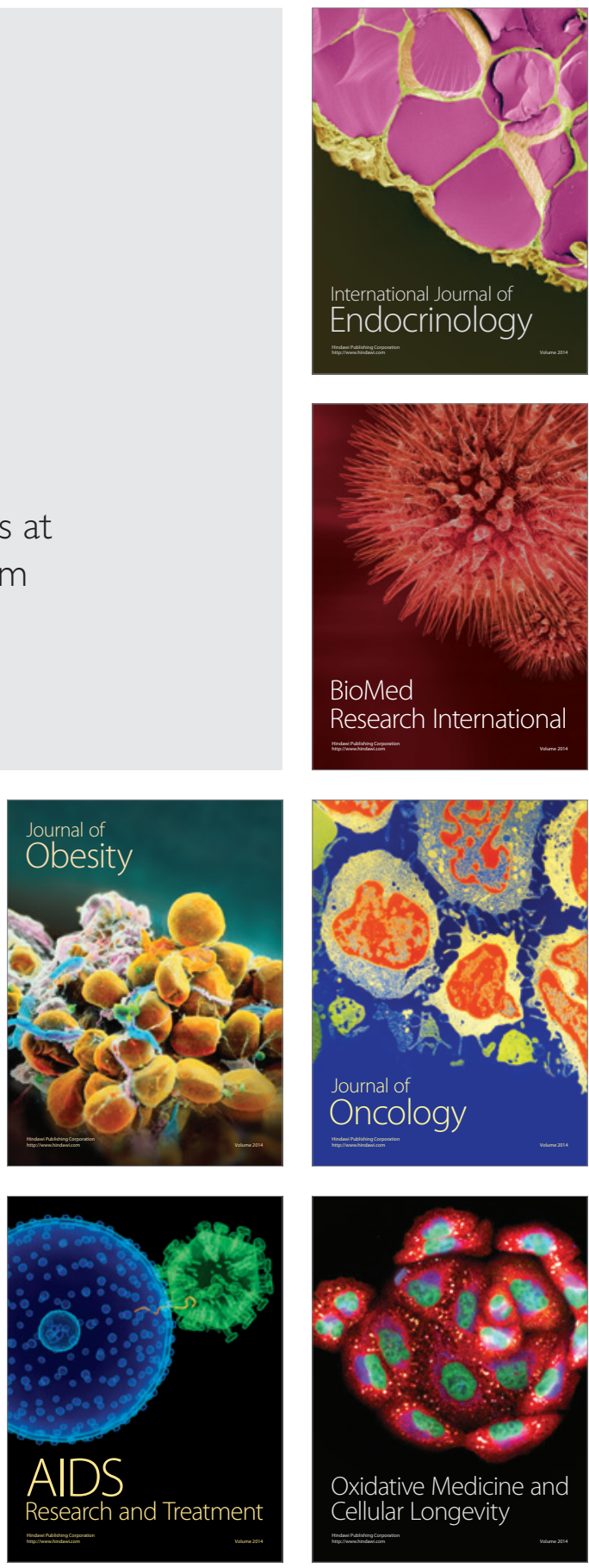